\title{
Data collection algorithm for wireless sensor networks using collaborative mobile elements
}

\author{
Alhasanat Abdullah ${ }^{1}$, Alhasanat Khaled ${ }^{2}$, Ahmed Maamoun ${ }^{3}$ \\ ${ }^{1}$ Department of Computer Engineering, Alhussein Bin Talal University, Jordan \\ ${ }^{2}$ Faculty of Information Technology, Middle East University, Jordan \\ ${ }^{3}$ Department of Computer Science, Aqaba University of Technology, Jordan
}

\begin{tabular}{l} 
Article Info \\
\hline Article history: \\
Received Nov 17, 2017 \\
Revised Dec 6, 2018 \\
Accepted Jan 2, 2019 \\
\hline
\end{tabular}

\section{Keywords:}

Collaborative mobile elements

Data gathering

Multiple mobile elements

Wireless sensor networks

\begin{abstract}
The simplest approach to reduce network latency for data gathering in wireless sensor networks (WSN) is to use multiple mobile elements rather than a single mobile sink. However, the most challneging issues faced this approach are firstly the high network cost as a result of using large number of mobile elements. Secondly, it suffers from the difficulty of network partitioning to achieve an efficient load balancing among these mobile elements. In this study, a collaborative data collection algorithm (CDCA) is developed. Simulation results presented in this paper demonstrated that with this algorithm the latency is significantly reduced at small number of mobile elements. Furthermore, the performance of CDCA algorithm is compared with the Area Splitting Algorithm (ASA). Consequently, the CDCA showed superior performance in terms of network latency, load balancing, and the required number of mobile elements.
\end{abstract}

Copyright () 2019 Institute of Advanced Engineering and Science. All rights reserved.

\section{Corresponding Author:}

Alhasanat Abdullah,

Department of Computer Engineering,

Alhussein Bin Talal University,

Maan, Jordan, P.O.Box 20.

Email: abad@ahu.edu.jo

\section{INTRODUCTION}

Indeed, using a single mobile element for data gathering in Wireless Sensor Networks (WSNs) leads to high latency, particularly for large sensor fields [1]. In addition, it is often difficult for a single mobile element to traverse long paths and collect data from all nodes due to the limited energy resources [2]. For these reasons, dozens of techniques have been emerged which introduced the concept of using several mobile elements for data gathering tasks [3]-[5]. With this approach, the path length of mobile elements is shortened which as a result leads to minimize the energy consumption and to reduce the overall data gathering latency. In spite of the benefits of using multiple mobile elements approach, it introduces additional cost to the network since the number of mobile elements involved in the data gathering task is increased. This represents unfeasible solution for many WSN applications [6]-[8]. The main challenging of this study is therefore to use the lowest possible number of mobile elements whilst minimizing data gathering latency.

In this paper, a new data gathering technique called Collaborative Data Collection Algorithm (CDCA), is introduced. The key idea of the proposed algorithm is to divide the network field into fixed partitions of equal areas. Then, each partition is allocated to a mobile element for which this mobile element traverses in a predetermined path and collects data from sensor nodes belong to its area and upload the collected data into a sink node. The path of the mobile element is designed such that, firstly, all nodes should be covered and secondly the path length of the mobile element should be satisfied with the path constraint which is usually given in advance. 
The benefits of using this algorithm are: firstly, the shortest path algorithm, such as Travel Sales Man Problem (TSP), is not required since the mobile element will be moving into a predetermined path designed to pass through communication range of every sensor node. Secondly, the CDCA algorithm is considered as location-unaware as mobile elements collect data from nodes on-fly and locations of sensor nodes do not need to be available in advance. Thirdly, CDCA is highly scalable algorithm as removing (or adding) nodes makes no effects on the path of the mobile element. Fourthly, since all mobile elements have identical path lengths, in addition to reduce the data gathering latency; it allows the mobile elements to provide a consistent data collection frequency to the sink node. Furthermore, as sensor nodes are typically distributed in a uniform random manner, all partitions are almost accommodating the same number of sensor nodes. This in turn provides fairly loads to the mobile elements in term of data buffering and power consumption.

This paper is organized as follows. The literature review is given in the next section. The problem statement is formulated in Section 3. In Section 4, the proposed method is described. The performance analysis and simulation results are discussed in Section 5. The conclusion is drawn in Section 6.

\section{LITERATURE REVIEW}

Recently, using multiple mobile elements for data gathering in WSN have became an important trend in literature, as it provided a straightforward approach to improve the network scalability, latency and throughput [2]-[29]. For example, to keep away from the data thrashing due to buffer spread out, author in [4] proposed the Partitioning-based Scheduling (PBS) algorithm to program the arrangements of mobile elements in a sensor set of connections. This approach divides the related mobile elements into two sub-problems. Then, all nodes are correspondingly divided into various parts within a group, and then the scheduling algorithm attempted to reduce the overhead of moving back across the distant nodes. Using MPP problem for the mobile elements to extend the life span of WSN instead of using the single path was also investigated. In [12] fixed $\mathrm{K}$ and adaptive $\mathrm{K}$ schemes planned numerous paths for the mobile element. These paths were designed in order to maintain stability on energy use for separate sensor nodes. As a result of this study, the multi-path can prolong the life time up to four times.

In fact, latency is the most important issue when concerning data collection with multiple mobile elements [7]-[29]. In fact, data collection latency is mainly influenced by the speed of the mobile element and length of tour for which the mobile element will traverse. In an earlier work [11], it moved to overcome this problem by reducing the tour length of the mobile entity based on whether the base station (BS) can move through the networks or not. However, in case when the BS cannot move, it identified some mobile elements (MEs) to collect data and returned it to the BS. Hence, three different procedures were proposed focusing on this problem. First and foremost is the area-splitting algorithm (ASA), which divides the networks into various parts, each mobile entity is used for gathering data from one of the stop points. Second method is Lloyd's beased algorithm which deals with sensor networks that are randomly organized. Third method is the Genetic based algorithm which is considered as an intelligent algorithm in the sense of utilizing mobile element tour optimization schemes.

Other research, such as [5], [10], [14], [17] and [24] have focused on obtaining optimal trajectories of multiple mobile elements. For example, in [24] the k-travelling salesperson with neighbor (k-TSPN) is introduced. In this method, each mobile element while moving in it is path can broadcast data to the sink in its appropriate position. Moreover, a study like [8], [13], [17], [21] and [26] endeavoured to tackle the latency problem whilst maintaining less power consumption. For instance, in [21] a cooperative data gathering hierarchy scheme has been proposed. In this algorithm, two kinds of mobile elements were suggested. First one called mobile collector (MC) which used to collect data from sensor nodes. The other one is called mobile relay (MR) which categorized data from various mobile collectors and transfers the collected data to the sink node. Similarly, the method presented in this research utilized the concept of the mobile collector to group data from nodes and transmit it to the sink node at appropriate time and locations, however without based on any mobile relays. This further leads to decrease the cost of network overhead in addition to reducing data gathering latency.

On the other hand, authors in [6], [7], [11], [15], [16] and [25] relied on using multiple mobile elements instead of one or a static sink. They proposed algorithms for which multiple mobile elements used to collect data while moving through prescribed paths. For example, authors in [6] suggested an algorithm which splits the network into two important areas. The first one is the concentric sphere of a deployed region and the other area is further divided into eight sub-areas. The mobile elements move along the diameter of the sphere and other two sinks move along the arc lines to gather the data packet from sensor nodes. It is demonstrated by the simulation results that this algorithm efficiently modified the hot spots trouble and prolonged the network life span of WSNs. In contrast, in this research the network is repeatedly divided to a 
number of horizontal and vertical partitions until the path length and communication range constraints of mobile elements are satisfied. The number of network partitions is therefore variant and independent on the number and locations of sensor nodes.

\section{PROBLEM FORMULATION}

Consider that a WSN consists of $N$ sensor nodes that are uniformly and randomly distributed within an area of $K x L$ meters square and $M$ mobile elements used to collect data. Supposing that all sensor nodes are using static transmission power, i.e. same level of transmission power and mobile elements are moved on predefined paths at a fixed speed. In each data gathering round, mobile elements are required to traverse its path whilst collecting data from nodes and then upload the collected data into the sink node. The sink node is supposed to be mounted at the origin point of the sensor field.

As mentioned in the introduction section, data gathering task with multiple mobile elements requires the satisfaction of the following conditions:

a. Covering all sensor nodes in the network field.

b. Satisfying path length constraint of the mobile element, such that

$$
l \leq C
$$

where $l$ is total path length of the mobile element and $C$ is path length constraint. The following table represents all mathematical notations used in this paper.

\section{THE PROPOSED METHOD}

In this section, the Collaborative Data Collection Algorithm (CDCA) is discussed. The algorithm attempts to reduce the network latency whilst maintaining the least number of mobile elements. The main idea behind the CDCA is to, as necessary, divide the network field into a number of partitions, for each partition a mobile element is allocated. The number of mobile elements that is required to cover all sensor nodes, i.e. satisfying the requirement of condition (1), is equivalently the same number of partitions in the horizontal direction which is given as,

$$
M_{x}=\left\lceil\frac{K}{3(r-\tau)}\right\rceil
$$

where $r$ is the communication range of the mobile element and $\tau$ represent the deficiency of the communication range of mobile elements as a result of environmental conditions, such as noise, obstacles and interference [30]. The value of $\tau$ should be chosen carefully in order to ensure that every sensor node will be covered within the communication range of at least one mobile element. It is also worth mentioning that Equation (2) is computed such that in addition to covering all sensor nodes, every mobile element is also connected to its direct neighbor of mobile elements. This thought is important since it allows cooperation between mobile elements in a sense that distant mobile elements can bypass its data to the sink node without the need to make direct contact with it. According to the network partitions defined in (2), the mobile element should move along the rectangular path arranged on the middle of the network area. Accordingly, the path length of the mobile element can be computed as

$$
l_{x}=2\left(\frac{K}{M_{x}}-(r-\tau)\right)
$$

Figure 1 shows the scenario of computing the path of the mobile element in the horizontal direction. However, the computed path may not meet the path constraint given in (1). In this case, the network is further divided in the vertical direction. It is clear from Figure 2 that the required number of partitions in the vertical direction is obtained as

$$
M_{y}=\left\lceil\frac{l_{x}+2 l-(r-\tau)}{C}\right\rceil
$$

Therefore, the mobile element will move horizontally along the path $l_{x}$, where

$$
l_{y}=2\left(\frac{1}{M_{y}}-(r-\tau)\right)
$$


The overall number of mobile elements that satisfies the above mentioned conditions, is given as

$$
M=M_{x} \times M_{y}
$$

Similarly, the total path length of a single mobile element is computed as

$$
l=l_{x} \times l_{y}
$$

The CDCA can be briefly described in the following three phases.

\subsection{Phase 1}

In this phase, the two conditions (i.e. covering all sensor nodes in the network field and satisfying the path constraint) are achieved. In this phase, to specify the number of mobile elements that is used to collect data from all sensor nodes, the network field is divided horizontally into sub-areas, for each sub-area a mobile element is assigned. Figure 1 illustrates an example of using three mobile elements to cover all the sensor nodes in the field. However, when the path length of a ME is greater than its constraint, the network area need to be further divided vertically until condition (1) is satisfied. Figure 2 represents the horizontal and vertical division of the network area.

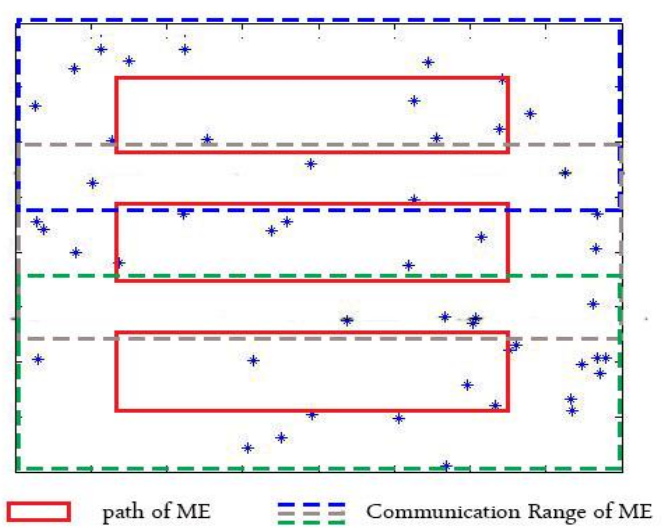

Figure 1. Horizontal division of the network area

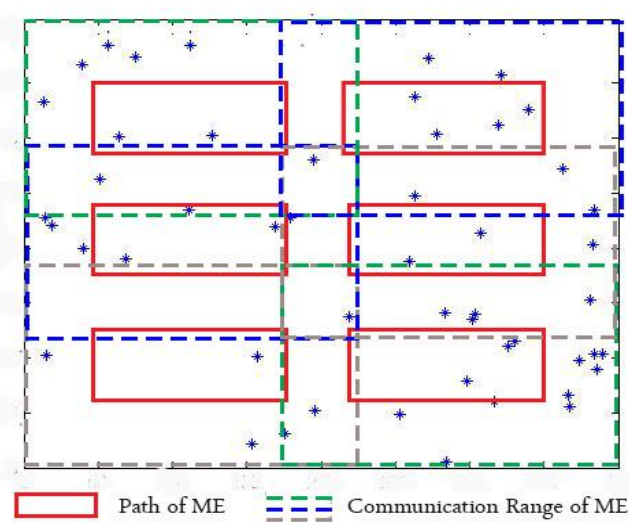

Figure 2. Horizontal and vertical division of the network area

\subsection{Phase II}

To allow cooperation between mobile elements, specifics startup times for every mobile element should be defined individually. Hence, assume that a mobile element starts its data collection round at $t_{0}$, therefore its neighbors of mobile element should start their collection round $t_{0}+t$. This allows two neighbors of MEs to be temporarily resided on the communication range of each other where the data collection cooperation takes place. Generally speaking, the startup time matrix of all mobile elements is given.

$$
T=\left[\begin{array}{ccc}
t_{1,1} & \cdots & t_{1, M_{y}} \\
\vdots & \ddots & \vdots \\
t_{M_{x, 1}} & \cdots & t_{M_{x}, M_{y}}
\end{array}\right]
$$

Hence, the data collection startup times for all mobile elements are defined as

$$
T=\left[\begin{array}{cccc}
t_{0} & t+t_{0} & t_{0} & t+t_{0} \\
t+t_{0} & t_{0} & t+t_{0} & t_{0} \\
t_{0} & t+t_{0} & t_{0} & t+t_{0} \\
t+t_{0} & t_{0} & t+t_{0} & t_{0}
\end{array}\right]
$$


where, $t=\frac{l}{2 s}$, and $l$ is the path length of the mobile element and $s$ is the speed of the mobile element. For Example, Figure 3(a) illustrates the locations of 3x3 mobile elements at the startup time. The locations of mobile elements at $t+t_{0}, t+t_{0}+\frac{l y}{4 s}, t+t_{0}+\frac{l}{s}-\frac{l x}{4 s}$ are shown in Figure $3(\mathrm{~b})$-(d), respectively. In addition, at Phase II, each sensor node knows which mobile element it should belong. Hence, at the initial round, while the mobile element traversing its path, the node selects the mobile element with the highest Received Signal Strength (RSS) value as its data collector. At the same time, it records the arriving time of that mobile element to compute its data collection time. For example, consider that $t_{c}^{0}$ is the very initial arrival time of a mobile element for a node, then the data collection time for this node at round $i$ is given as

$$
t_{c}^{i}=t_{c}^{0}+\frac{i l}{s}
$$

In fact, the knowledge of collection times is also important for sensor nodes as these nodes will be aware of inactive intervals. Consequently, they can enter the sleep mode between inter-collection times in order to save their energy resources.

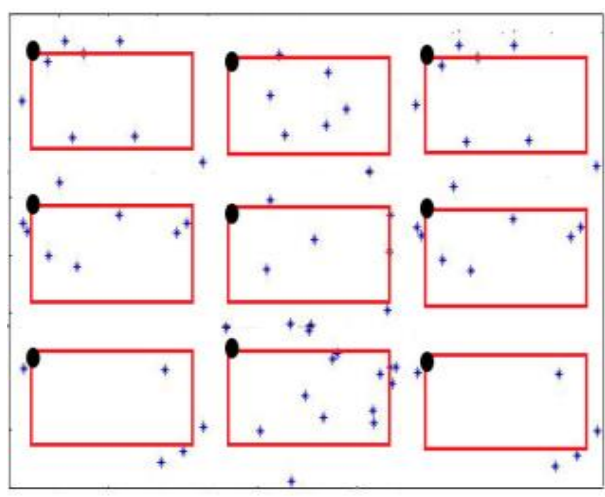

(a)

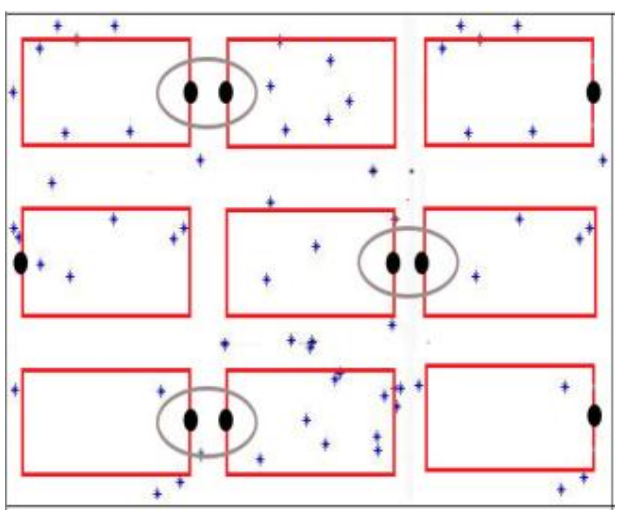

(c)

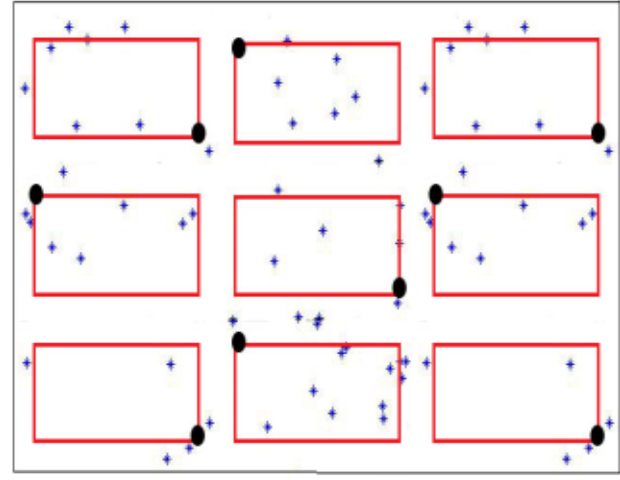

(b)

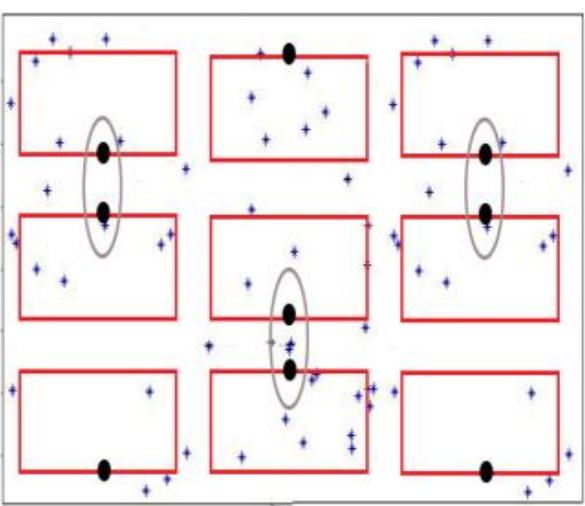

(d)

Figure 3. Mobile Elements locations at (a) $t_{0}$, (b) $t+t_{0}$, (c) $t+t_{0}+\frac{l y}{4 s}$ and (d) $t+t_{0}+\frac{l}{s}-\frac{l x}{4 s}$

\subsection{Phase III}

In this phase, data collection is taken place. Each mobile element starts collecting data from all nodes in its partition and then sends this data to the closest mobile element, in order to be delivered to the sink node. Figure 4 illustrates the flowchart of the CDCA algorithm. 


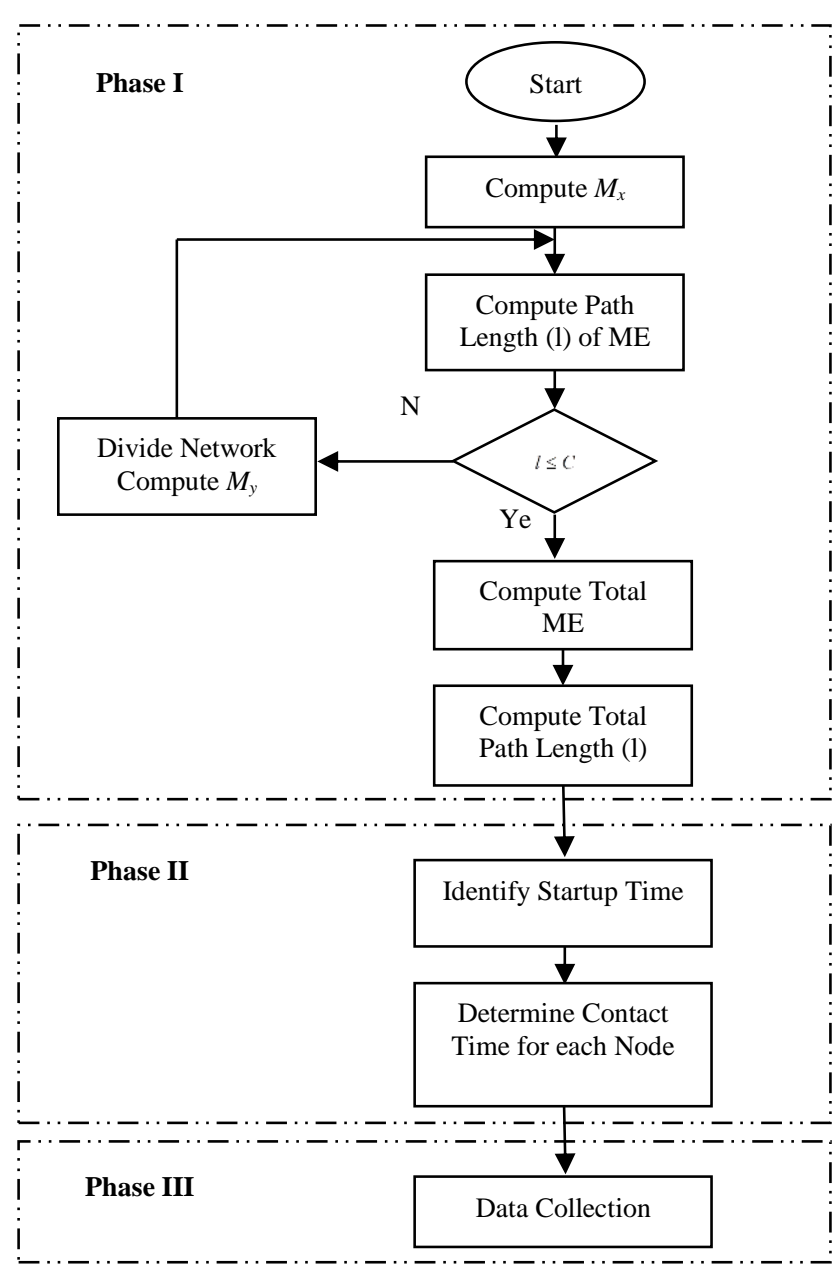

Figure 4. The flowchart of the CDCA algorithm

\section{RESULTS AND ANALYSIS}

In this section, the performance of the CDCA algorithm is evaluated. The CDCA algorithm is also compared with the Area Splitting Algorithm (ASA) [8]. The efficiency of these algorithms is compared in terms of a number of mobile elements, load balancing and data gathering latency.

In this experiment, a set of sensor nodes are uniformly and randomly deployed within a two dimensional square sensor field. The sink node is located at the origin point of the sensor field. The mobile element speed is set to $1 \mathrm{~m} / \mathrm{s}$. The mobile element is designed to move through a predefined route from a point and returns to the same point to collect data from sensor nodes using single hop communication. An average of 200 independent runs of this experiment is computed using Matlab Monte-Carlo simulation. Table 1 represents all simulation parameters used in this experiment.

Table 2. The Simulation Parameters used in this Paper

\begin{tabular}{ccc}
\hline Parameter & Value & Description \\
\hline $\mathrm{R}$ & $40 \mathrm{~m}$ & Communication Range \\
$\mathrm{K}, \mathrm{L}$ & $300 \times 300$ & Network Area \\
$\mathrm{N}$ & 100 & Node Number \\
$\tau$ & $2 \mathrm{~m}$ & Range deficiency \\
$\mathrm{C}$ & $800 \mathrm{~m}$ & Path Constraint \\
\hline
\end{tabular}

\subsection{The number of mobile elements}

As mentioned before, one of the main goals of this paper is to perform data gathering task with the minimum number of mobile elements. To this end, the number of mobile elements used for the two algorithms, CDCA and ASA, as a function of increasing network size and path constraints is shown in 
Figure 5(a) and Figure 5(b), respectively. In Figure 5(a), the number of mobile elements is rapidly growing as the network area enlarged. The CDCA algorithm, in contrast, shows a slight change of the number of mobile elements which led to reduce the network cost significantly. Similarly, in Figure 5(b), the CDCA algorithm required less number of mobile elements in comparison with the ASA, particularly at short path constraint.

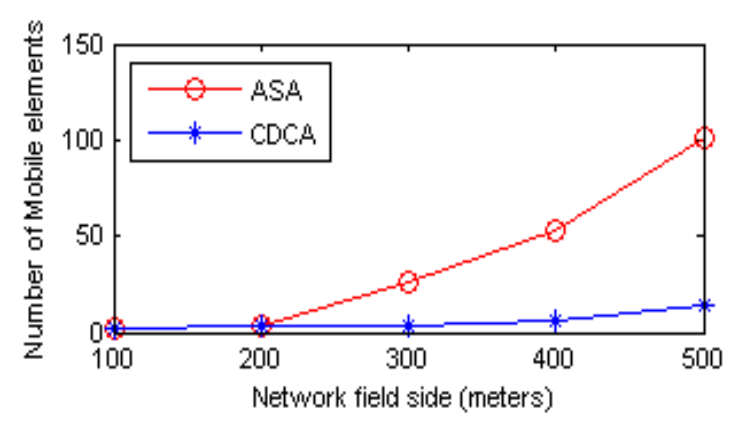

(a)

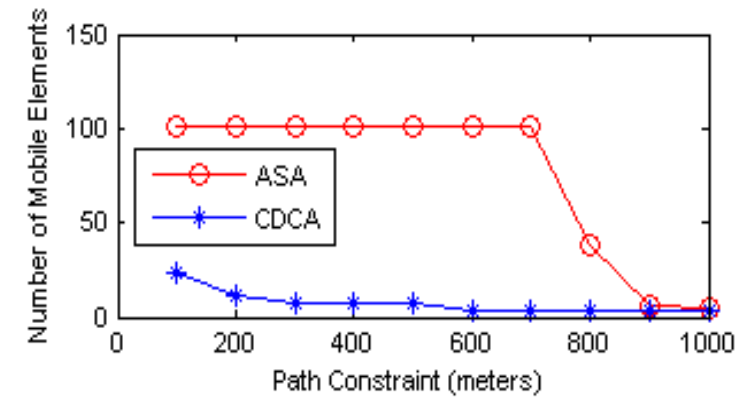

(b)

Figure 5. The number of mobile elements as a function of (a) increasing network areas, and (b) increasing path constraints of mobile elements

\subsection{Load balancing on mobile element}

The load balancing of multiple mobile elements is one of important consideration when designing an efficient data gathering algorithm. By attaining this goal, the network latency will be reduced in addition to better usage of the mobile element resources such as power and buffer size. The most common factor used to measure this criterion is the Variation Coefficient (VC). VC is defined as the percentage of standard deviation of tour lengths of all mobile elements and their mean value [6]. In our case, in the CDCA algorithm all mobile elements will traverse identical path lengths and therefore the VC will be almost zero. For a fairness and consistent comparison, the VC is computed based on the number of sensor nodes assigned to each mobile element, which can be written as:

$$
V C \%=\frac{\sum_{i=1}^{M} \sigma_{i}}{\frac{1}{M} \sum_{i=1}^{M} N_{i}} 100 \%
$$

where $\sigma_{\mathrm{i}}$ is the standard deviation of the number of sensor nodes $N_{i}$ belonging to mobile element $i$, and $M$ is the total number of mobile elements. Note that the lower the variation coefficient (VC), the better the load balance for each mobile element. Figure 6(a) illustrates the $V C$ for CDCA and ASA algorithms, at varying network areas. The $V C$ of the two algorithms is almost similar at small network areas. Despite this, as the network area increases, the performance gap between the two algorithms is expanded. This result is apparent since the CDCA algorithm demonstrates a consistent increase in the $V C$, whereas this value for the ASA algorithm rapidly increases. Figure 6(b) shows the $V C$ as a function of increasing density of sensor nodes. Surprisingly, the $V C$ of the CDCA algorithm decreases as the number of sensor nodes increases, whereas this value is substantially increased for ASA algorithm. This emphasizes the efficiency of using CDCA algorithm for dense sensor networks. Similarly, Figure 6(c) shows the $V C$ obtained by the two algorithms. The load balance is improved for both algorithms, but the CDCA algorithm has lower $V C$ percentage than ASA over the entire range of path constraint. 


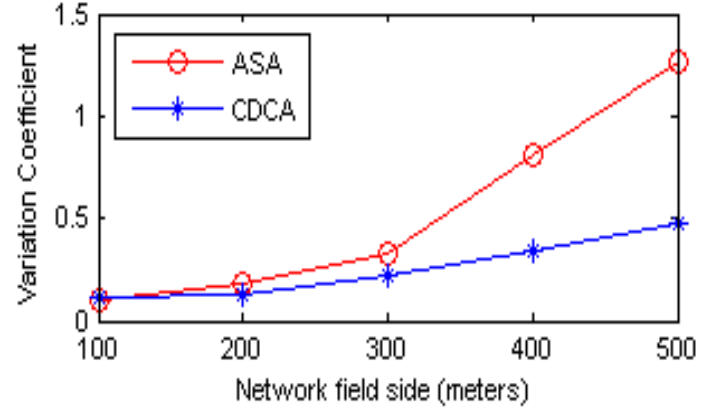

(a)

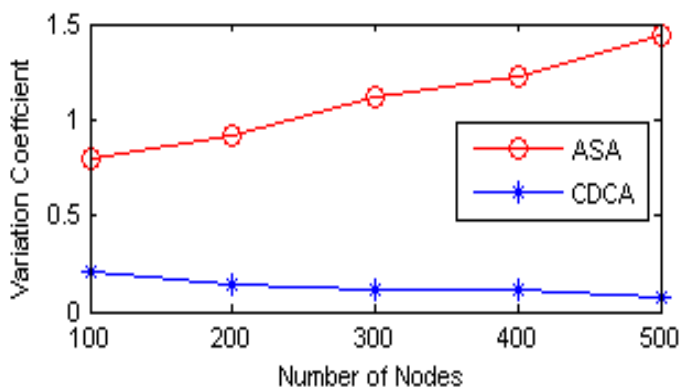

(b)

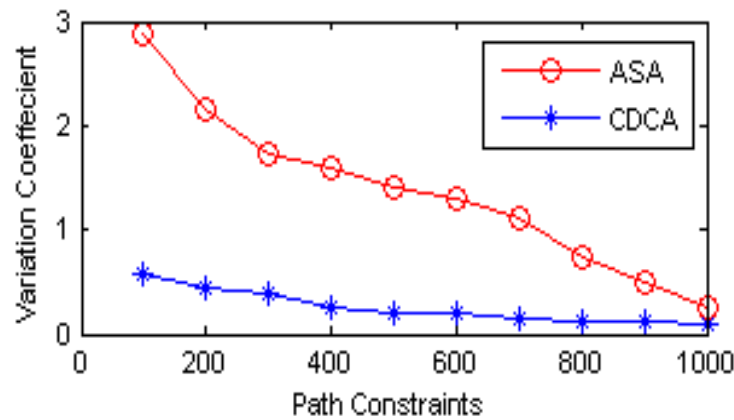

(c)

Figure 6. Variation Coefficient (VC) as a function of (a) Increasing Network Area, (b) Increasing Number of Sensor Nodes, (c) Increasing Path Constraints

\subsection{Network latency}

Latency can be defined as the duration of time required to complete one round of data gathering by mobile elements. As mentioned before, the increasing of mobile elements leads to decrease the network latency [8].

Figure 7 investigates the impact of network areas, number of nodes and the path constraint on the network latency for both algorithms. Figure 7(a) shows a gradual increase of the network latency for the two algorithms as the network area is enlarged with superiority to the CDCA algorithm. Moreover, in Figure 7(b) the latency is not changed with the increasing number of sensor nodes for the CDCA. This is not the case for the ASA algorithm, in which the network latency is slightly increased as a function of increasing the number of sensor nodes. This is due to the fact that the ASA algorithm used the Travel Sales-Man (TSP) algorithm to find the paths of mobile elements.

On the other hand, although long paths of mobile elements generally yield to reduce the number of mobile elements, the network latency is increased. This trend is shown in Figure 7(c). In this regard, the ASA algorithm exhibited an almost constant latency. Nevertheless, the CDCA algorithm accomplished the data gathering with lower latency over the entire range of the path constraints. In this figure, at low path constraints, the CDCA reduces the network latency substantially. 


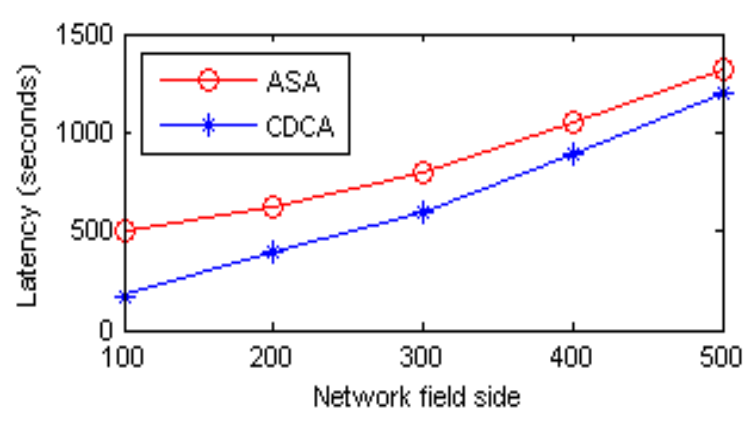

(a)

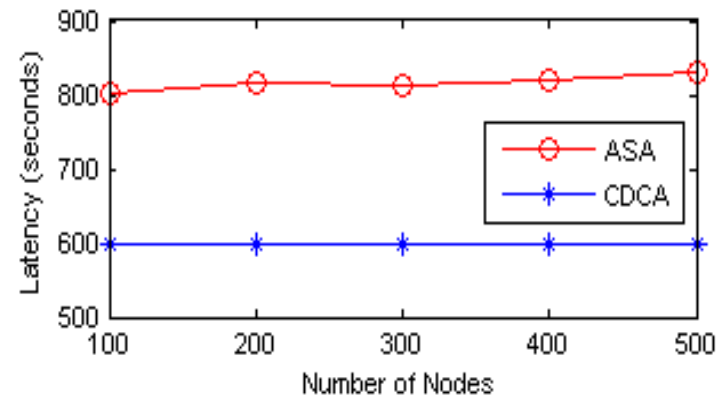

(b)

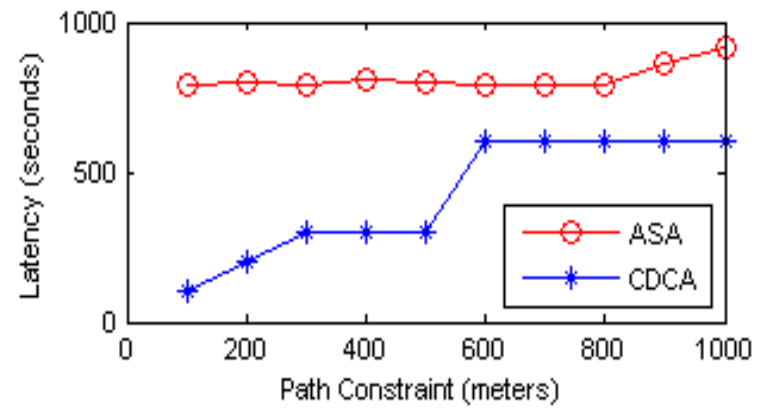

(c)

Figure 7. Network latency as a function of (a) increasing network area,

(b) increasing number of sensor nodes, (c) increasing path constraints

\section{CONCLUSION}

In this paper a new data gathering technique in WSN based on using more than one mobile element, referred to as collaborative data collection algorithm (CDCA), was proposed. The main objective behind the CDCA is to reduce data gathering latency which in turn leads to increase the network throughput and to decrease the number of mobile elements used for this task. Simulation results showed that the CDCA exhibited superior performance in comparison with the ASA algorithm in term of load balancing, number of mobile elements and network latency.

In future work, as each mobile element is designed to collect data from large number of sensor nodes, the buffer size limitation could be considered. In addition, it would be worthwhile to study the way of delivering data through mobile elements to the sink node. It would also be useful to further study the power recharging cycles of the mobile elements from temporal and spaial perspective.

\section{REFERENCES}

[1] W. B. Heinzelman, et al., "An application-specific protocol architecture for wireless microsensor networks," IEEE Transactions on Wireless Communications, vol/issue: 1(4), pp. 660-670, 2002.

[2] K. Dantu, et al., "Robomote: Enabling mobility in sensor networks," Information Processing in Sensor Networks, IPSN, Fourth International Symposium, pp. 404-409, 2005.

[3] J. Wang, et al., "Multiple mobile sink-based routing algorithms for data dissemination in wireless sensor networks," concurrency and computation: practice and experience, Wiley Online Library, vol/issue: 27(10), pp. 2656-2667, 2015.

[4] Y. Gu, et al., "Partitioning Based Mobile Element Scheduling in Wireless Sensor Networks," Proc. of SECON, Santa Clara, U.S., pp. 386-395, 2005.

[5] B. Yuan, et al., "On the optimal robot routing problem in wireless sensor networks," IEEE Trans. Knowl. Data Eng., vol/issue: 19(9), pp. 1252-1261, 2007.

[6] G. Xing, et al., "Rendezvous planning in wireless sensor networks with mobile elements," IEEE Transactions on Mobile Computing, vol/issue: 7(12), pp. 1430-1443, 2008.

[7] D. Zhu, et al., "Multi-path Planning for Mobile Element to Prolong the Lifetime of Wireless Sensor Networks," 2009 15th IEEE International Conference on Embedded and Real-Time Computing Systems and Applications, Beijing, pp. 41-50, 2009. 
[8] E. C. H. Ngai, et al., "An Adaptive Delay-Minimized Route Design for Wireless Sensor-Actuator Networks," IEEE Transactions on Vehicular Technology, vol/issue: 58(9), pp. 5083-5094, 2009.

[9] S. Gao and H. Zhang, "Energy Efficient Path-constrained Sink Navigation in Delay- guaranteed Wireless Sensor Networks," Journal of Networks, vol/issue: 5(6), pp. 658-665, 2010.

[10] S. Gao, et al., "Efficient Data Collection in Wireless Sensor Networks with Path-Constrained Mobile Sinks," IEEE Transactions on Mobile Computing, vol/issue: 10(5), pp. 592-608, 2011.

[11] L. He, et al., "Analysis on data collection with multiple mobile elements in wireless sensor networks," Global Telecommunications Conference (GLOBECOM 2011), IEEE, pp. 1-5, 2011.

[12] W. Kwong and Y. Xu, "Path planning algorithm for space manipulator with a minimum energy demand," Robotics and Biomimetics (ROBIO) 2012 IEEE International Conference on, pp. 1556-1563, 2012.

[13] X. Xu, et al., "A Delay-Efficient Algorithm for Data Aggregation in Multihop Wireless Sensor Networks," IEEE Transactions on Parallel Distributed Systems, vol/issue: 22(1), pp. 163-175, 2011.

[14] G. Xing, et al., "Efficient rendezvous algorithms for mobility-enabled wireless sensor networks," IEEE Transactions on Mobile Computing, vol/issue: 11(1), pp. 47-60, 2012.

[15] L. He, et al., "A Progressive Approach to Reducing Data Collection Latency in Wireless Sensor Networks with Mobile Elements," IEEE Transactions On Mobile Computing, vol/issue: 12(7), pp. 1308-1320, 2013.

[16] M. Ma, et al., "Tour Planning for Mobile Data-Gathering Mechanisms in Wireless Sensor Networks," IEEE Transactions on Vehicular Technology, vol/issue: 62(4), pp. 1472-1483, 2013.

[17] D. Kim, et al., "Minimum Latency Multiple Data MULE Trajectory Planning in Wireless Sensor Networks," IEEE Transactions on Mobile Computing, vol/issue: 13(4), pp. 838-851, 2014.

[18] J. S. He, et al., "Constructing Load-Balanced Data Aggregation Trees in Probabilistic Wireless Sensor Networks," IEEE Transactions on Parallel and Distributed Systems, vol/issue: 25(7), pp. 1681-1690, 2014.

[19] D. Gong and Y. Yang, "Low-latency SINR-based data gathering in wireless sensor networks," IEEE Trans. Wireless Commun., vol/issue: 13(6), pp. 3207-322, 2014.

[20] H. Lin and H. Üstert, "Exact and heuristic algorithms for data-gathering cluster based wireless sensor network design problem," IEEE/ACM Transactions on Networking, vol/issue: 22(3), pp. 903-916, 2014.

[21] D. V. Le, et al., "HiCoDG: A hierarchical data-gathering scheme using cooperative multiple mobile elements," Sensors, vol/issue: 14(12), pp. 24278-24304, 2014.

[22] M. Zhao, et al., "Mobile Data Gathering with Load Balanced Clustering and Dual Data Uploading in Wireless Sensor Networks," IEEE Transactions On Mobile Computing, vol/issue: 14(4), pp. 770-785, 2015.

[23] Y. Yao, et al., "EDAL: An energy-efficient, delay-aware, and lifetime-balancing data collection protocol for heterogeneous wireless sensor networks," IEEE/ACM Transactions on Networking, vol/issue: 23(3), pp. 810-823, 2015.

[24] C. F. Cheng and C. F. Yu, "Data Gathering in Wireless Sensor Networks: A CombineTSPReduce Approach," IEEE Transactions on Vehicular Technology, vol/issue: 65(4), 2309-2324, 2016.

[25] D. Kim, et al., "Minimizing data collection latency in wireless sensor network with multiple mobile elements," 2012 proceeding INFOCOM, IEEE, pp. 504-512, 2012.

[26] J. He, et al., "Delay Minimization for Data Dissemination in Large-Scale VANETs with Buses and Taxis," IEEE Transactions on Mobile Computing, vol. 15, pp. 1939-1950, 2016.

[27] D. Gosain and I. Snigdh, "Performance Comparison of Routing Protocols in Bipartite Wireless Sensor Network," International Journal of Electrical and Computer Engineering, vol/issue: 5(6), pp. 1417-1423, 2015.

[28] S. Umar, et al., "Tree Based Energy Balancing Routing Protocol by Self Organizing in Wireless Sensor Networks," International Journal of Electrical and Computer Engineering, vol/issue: 5(6), pp. 1486-1491, 2015.

[29] K. N. Qureshi, et al., "Geographical Forwarding Methods in Vehicular Ad hoc Networks," International Journal of Electrical and Computer Engineering, vol/issue: 5(6), pp. 1407-1416, 2015.

[30] M. F. Iskander and Z. Yun, "Propagation prediction models for wireless communication systems," IEEE Transactions on Microwave Theory and Techniques, vol/issue: 50(3), pp. 662-673, 2002. 